\title{
A Tailored Habits-based Dietary Intervention Combined With Oral Rehabilitation on Partially Dentate Older Adults: Nutritional Impact
}

\begin{abstract}
Introduction: Replacing missing teeth alone is not enough to engender dietary behaviour change amongst older adults. Whilst there is a body of evidence to support oral rehabilitation in conjunction with dietary advice, this is currently limited to edentate patients even though the majority of older adults are now partially dentate. One approach proven to change long-term food behaviours but is novel in this population is habit-formation. Consequently, this study developed and tested a habit-based tailored dietary intervention, in conjunction with oral rehabilitation amongst partially dentate older adults.
\end{abstract}

Materials and methods: A pilot randomised control trial was conducted on 57 partially dentate older patients. Participants were randomised to an intervention group (habits-based dietary intervention) or a control group and followed up for 8 months. The intervention group attended four meetings with a trained researcher to target habit-formation around 3 dietary domains (fruit/vegetables, wholegrains, healthy proteins). The primary outcome measure was self-reported automaticity for developing healthy habits and habit formation was assessed using the Self-Report Behavioural Automaticity Index (SRBAI). Preliminary analysis was conducted on $\mathrm{n}=36$ participants between baseline and 8 month follow up.

Results: Preliminary results showed that SRBAI scores and self-reported frequency of days doing habits in the intervention group for all tailored dietary habits was significant between baseline and follow up visits $(p<0.001)$. There were moderate positive correlations between automaticity and habit adherence (Fruit/vegetables rho $=0.43, p=0.09$ : Wholegrains rho $=0.44, p=0.08$ : Healthy Proteins rho $=0.52, p=0.03)$ for the intervention group. Automaticity trends were increased in the intervention group for all 3 dietary habits compared to the control group but, other than wholegrain $(p=0.005)$, between group differences were non-significant $(p>0.05)$. BMI decreased in the intervention group $\left(29.6\right.$ to $\left.28.7 \mathrm{~kg} / \mathrm{m}^{2}\right)$ compared to a non-significant increase in the control group $\left(27.7\right.$ to $\left.27.8 \mathrm{~kg} / \mathrm{m}^{2}\right)$ $(\mathrm{p}=0.08)$. There were slight increases in Mini Nutritional Assessment mean change scores $(0.19$ intervention: 0.32 control $)$ for both groups, however between-group differences were not statistically significant $(p=0.9)$. Greater improvements in food intake around dietary habits were observed in the intervention group (Fruit/vegetables:108 g Fibre 4g: Protein 11g) compared to the control group (Fruit/vegetables $-17 \mathrm{~g}$ : Fibre 2g: Protein $-4 \mathrm{~g}$ ).

Discussion: Preliminary results demonstrate the success of a habit-based dietary intervention coupled with oral rehabilitation in positively influencing dietary behaviours and other nutritional outcomes in partially dentate older adults.

\section{Conflict of Interest}

There is no conflict of interest 